Canad. Math. Bull. Vol. 23 (1), 1980

\title{
NOTES ON FRATTINI SUBGROUPS OF GENERALIZED FREE PRODUCTS WITH CYCLIC AMALGAMATION
}

\author{
BY
}

\author{
R. B. J. T. ALLENBY, C. Y. TANG ${ }^{(1)}$ AND S. Y. TANG
}

1. The problem of the exact location of the Frattini subgroup $\Phi(G)$ of a generalized free product $G=\left(A^{*} B\right)_{H}$ was first raised by Higman and Neumann [5]. Solutions to special cases of the problem can be found in [1], [2], [8], [9] and [10]. The purpose of this note is to extend the results of [2], [8], and to simplify the proof of Whittemore's theorem [10]. We also apply our result to give simple proofs of certain classes of knot groups that have trivial Frattini subgroups. The proof that every knot group has trivial Frattini subgroup hard and long (footnote 2, p. 56).

2. Whittemore's theorem. In [10] Whittemore showed that if $G=\left(A^{*} B\right)_{\mathbf{H}}$ contains an element $c$ such that $H \cap H^{c}=1$ then $\Phi(G)=1$. Applying this result it was shown that the Frattini subgroup of a generalized free product of finitely many finitely generated free groups amalgamating a cyclic subgroup is trivial. From this it follows immediately that the surface groups and the non-degenerated Fuchsian groups have trivial Frattini subgroups. However the proof of Whittemore's theorem is quite long and tedious to follow. Here we shall present a more understandable proof.

TheOREM $2.1([10])$. Let $G=\left(A^{*} B\right)_{H}$. If $G$ contains an element $c$ such that $H \cap H^{c}=\{1\}$ then $\Phi(G)=\{1\}$.

Proof. $H \cap H^{c}=\{1\}$ implies $H$ contains no nontrivial normal subgroups of $G$. Thus if $H$ has index 2 in both $A$ and $B$ we deduce $H=\{1\}$. It follows that $G$ is the ordinary free product of $A$ and $B$. That $\Phi(G)=\{1\}$ then follows from [5]. So WLOG we may assume $|A: H|>2$.

Now $\Phi(G) \triangleleft G$ and so if $\Phi(G) \leq H$ then again $\Phi(G)=\{1\}$. Thus we may also assume there exists $g \in \Phi(G) \backslash H$. Suppose $g$ has the normal form $g=$ $a_{1} b_{1} \cdots a_{n} b_{n}$ where $a_{i} \in A \backslash H, b_{i} \in B \backslash H$. Since $|A: H| \geq 3$ there exists $a \in A \backslash H$ such that $a^{-1} a_{1} \in A \backslash H$. Thus $g^{a}=\left(a^{-1} a_{1}\right) b_{1} \cdots a_{n} b_{n} a$ is an element of $\Phi(G)$ having normal form with initial and final letters both in $A \backslash H$. Consequently it is clear that any $g$ in $\Phi(G) \backslash H$ has a conjugate (also in $\Phi(G)$ ) having normal

Received by the editors October 25, 1977.

(1) The work of the second author was partly supported by a grant from the Canadian National Research Council No. A-4064. 
form with initial and final letters both in $A \backslash H$. WLOG we may assume $g$ itself is of this form.

Now set $z=g^{x c y}$ where we choose $x=1$ or $x \in B \backslash H$ according as the initial letter of (a normal form of) $c$ is in $B \backslash H$ or $A \backslash H$ (clearly c cannot belong to $H$ ) and we choose $y=1$ or $y \in B \backslash H$ according as the final letter of $c$ is in $B \backslash H$ or $A \backslash H$. Choose $u \in A \backslash H$ arbitrarily. We show that $z \notin\left\langle A^{z}, B^{u}\right\rangle$. Suppose the contrary and let

$$
z=b_{0}^{u} a_{1}^{z} b_{1}^{u} \cdots a_{n}^{z} b_{n}^{u}
$$

where $n$ is chosen as small as possible. (We admit the possibility that either or both of $b_{0}, b_{n}$ may be 1). By the very forms of $z$ and $b_{0}^{u}$ it is clear that $z=b_{0}^{u}$ is impossible. Thus $n \geq 1$. In greater detail (2.1) is

$$
\begin{gathered}
z=y^{-1} c^{-1}\left(c y \cdot u^{-1} b_{0} u \cdot y^{-1} c^{-1}\right) x^{-1} g^{-1} x\left(c y \cdot a_{1} \cdot y^{-1} c^{-1}\right) \\
x^{-1} g x\left(c y \cdot u^{-1} b_{1} \cdot u \cdot \cdots\right) \cdots
\end{gathered}
$$

For $i=1,2, \ldots, n$ we have $c y a_{i} y^{-1} c^{-1} \notin H$. For otherwise, we have $y a_{i} y^{-1} \in H^{c}$ (directly) and $y a_{i} y^{-1} \in H$ (from considering the normal form of $c y a_{i} y^{-1} c^{-1}$ ). Hence $y a_{i} y^{-1} \in H \cap H^{c}$ and $a_{i}=1$ follows. Consequently $b_{i-1}^{u} a_{i}^{z} b_{i}^{u}$ reduces to $\left(b_{i-1} b_{i}\right)^{u}$ and we have a new "smaller" representation of $z$ than that given in (2.1). Similar arguments apply to $c y u^{-1} b_{i} u y^{-1} c^{-1}$ if $i=1, \ldots, n-1$.

Thus the (normal form) length of the R.H.S. of (2.1) is at least $l\left(y^{-1} c^{-1} x^{-1} g^{-1} x\right)+l\left(x^{-1} g x c y\right)$ which is evidently greater than the length $l(z)$ of $z$, an obvious contradiction. Thus $z \notin\left\langle A^{z}, B^{u}\right\rangle$, and $z \notin \Phi(G)$ follows (since $\left.\left\langle A^{z}, B^{u}, z\right\rangle=G\right)$. Thus $g \notin \Phi(G)$ and $\Phi(G)=\{1\}$ follows.

3. Cyclic amalgamation. In [2] it was shown that if $A, B$ are locally solvable and $H$ is infinite cyclic then $\Phi(G) \leq H$, where $G=\left(A^{*} B\right)_{H}$. The proof is quite long and involves tedious case analysis. Here, by applying Theorem 2.1 , we prove that any generalized free product with cyclic amalgamation has its Frattini subgroup contained in the amalgamated subgroup by a remarkably elementary and brief argument.

TheOrem 3.1. Let $G=\left(A^{*} B\right)_{H}$ where $H$ is cyclic. Then $\Phi(G) \leq H$.

Proof. In view of Theorem 2.9 [8] we can assume $H$ to be infinite cyclic. Suppose $\Phi(G) \not H$. It follows immediately from Theorem 2.1 [9] (see proof of Theorem 2.1 of [1]) that we may assume, without loss of generality the existence of $a \in A \cap \Phi(G)$ such that $a \notin H$. Choose $b \in B \backslash H$ arbitrarily and set $c=b a b^{-1} a^{-1}$. Clearly $c \in \Phi(G)$. Consider $X=\left\langle A^{c^{2}}, B\right\rangle$. We shall show $c \notin X$, hence $c \notin \Phi(G)$. This contradiction shows that $\Phi(G) \leq h$.

If $c \in X$ we can write

$$
c=b_{0} \cdot c^{-1} c^{-1} a_{1} c c b_{1} c^{-1} c^{-1} \cdots b_{n}
$$

with $a_{i} \in A, b_{i} \in B$ and $n$ minimal. 
By Theorem 2.1, we can assume $H^{a} \cap H \neq\{1\} \neq H^{b} \cap H$. In particular there exist integers $l, m, r, s$ such that,

$$
a^{-1} h^{l} a=h^{m}
$$

and

$$
b^{-1} h^{r} b=h^{s}
$$

where

$$
H^{a} \cap H=\left\langle h^{m}\right\rangle, \quad H^{b} \cap H=\left\langle h^{s}\right\rangle .
$$

By the length argument, for (3.1) to hold, we must have $c^{-1} a_{1} c \in H$. This implies that $a_{1}, a_{1}^{b}, a_{1}^{b a}, a_{1}^{b a b-1} \in H$. Let $a_{1}=h^{k}$. For $a_{1}^{b} \in H$, we must have

$$
b^{-1} a_{1} b=b^{-1} h^{k} b \in H \cap H^{b} \text {. }
$$

Thus, by (3.3), we must have,

$$
b^{-1} h^{k} b=h^{\alpha s}
$$

For some integer $\alpha$. Since $a_{1}^{b a} \in H$, it follows that $a^{-1} h^{\alpha s} a \in H \cap H^{a}$. Therefore, by (3.2), there must exist an integer $\beta$ such that,

$$
\alpha s=\beta l .
$$

Hence,

$$
a^{-1} h^{\alpha s} a=a^{-1} h^{\beta l} a=h^{\beta m} .
$$

Next $b h^{\beta m} b^{-1}=a_{1}^{b a b-1} \in H$. Therefore, by (3.3), we must have $h^{\beta m} \in H \cap H^{b}$. Hence there exists an integer $\gamma$ such that

$$
\beta m=\gamma s \text {. }
$$

Thus, again by (3.3), $b h^{\beta m} b^{-1}=h^{\gamma r}$. Finally $a h^{\gamma r} a^{-1}=a_{1}^{c} \in H$ implies $h^{\gamma r} \in$ $H \cap H^{a}$. Hence, as before, there must exist an integer $\delta$ such that,

$$
\gamma r=\delta m \text {. }
$$

It follows that $c^{-1} a_{1} c=a h^{\delta m} a^{-1}=h^{\delta l}$. Now by (3.6) and (3.7) we have $\beta m r=$ $\gamma s r=\delta m s$. Hence,

$$
\beta r=\delta s \text {. }
$$

On the other hand, by (3.5) and (3.8) we have $\alpha s r=\beta l r=\delta s l$. Therefore $\alpha r=\delta l$. Thus $b h^{\alpha s} b^{-1}=h^{\alpha r}=h^{\delta l}$, whilst, by (3.4), $b h^{\alpha s} b^{-1}=h^{k}$. Hence $\delta l=\alpha r=$ $k$. It follows that,

$$
c^{-1} a_{1} c=c^{-1} h^{k} c=h^{\delta l}=h^{k} .
$$

This means $c$ commutes with $a_{1}$, whence $c^{-2} a_{1} c^{2}=a_{1} \in H$. Therefore we can write (3.1) as:

$$
c=\left(b_{0} a_{1} b_{1}\right) a_{2}^{c^{2}} \cdots b_{n}
$$


Thus (3.9) contradicts the minimal choice of $n$. Hence $c^{-1} a_{1} c \notin H$. In the same way, we can argue $c^{-1} a_{i} c \notin H$ and $c b_{i} c^{-1} \notin H$ for all $i$. It follows that the R.H.S. of (3.1) in its reduced form has length greater than $c$. This says $c \notin X$, whilst $G=\langle X, c\rangle$. Hence $c \notin \Phi(G)$ which in turn implies $a \notin \Phi(G)$ contradicting the assumption $a \in A \cap \Phi(G)$ such that $a \notin H$. Thus $a \in H$. Hence $\Phi(G) \subseteq H$. This proves the theorem.

4. A, B finitely generated and $\Phi$-free. In [8] it was shown that if $H$ is a finite cycle and if $N$ is the maximal $G$-normal subgroup of $H$ then $\Phi(G)=$ $\langle\Phi(A) \cap N, \Phi(B) \cap N\rangle$. We give an example to show that we cannot obtain such a result if $H$ is an infinite cycle.

EXAMPLE 4.1. Let

$$
A=\left\langle a, x, y ;[x, y]=1, x^{a}=x y^{2}, y^{a}=y\right\rangle
$$

and $B=\langle z\rangle$. Then it follows easily that $\left\langle y^{2}\right\rangle$ is the Frattini subgroup of $A$. Set $H=\langle y\rangle=\left\langle z^{2}\right\rangle$ and set $G=\left(A^{*} B\right)_{\mathrm{H}}$. Then $\Phi(G)=H$. (Map $G$ to $G /\left\langle y^{2}\right\rangle=A /\left\langle y^{2}\right\rangle * B /\left\langle y^{2}\right\rangle$ with $H /\left\langle y^{2}\right\rangle$ amalgamated and observe that

$$
\Phi\left(G /\left\langle y^{2}\right\rangle\right) \subseteq\langle y\rangle /\left\langle y^{2}\right\rangle=\Phi\left(B /\left\langle y^{2}\right\rangle\right) \subseteq \Phi\left(G /\left\langle y^{2}\right\rangle\right),
$$

since $\langle y\rangle\left\langle\left\langle y^{2}\right\rangle\right.$ is a finitely generated normal subgroup of $G /\left\langle y^{2}\right\rangle$. Now take inverse images to get back into $G$.) Thus $\langle\Phi(A) \cap H, \Phi(B) \cap H\rangle=\left\langle y^{2}\right\rangle \varsubsetneqq \Phi(G)$.

In [8] the second author gave an example of a generalized free product $G=\left(A^{*} B\right)_{H}$ where $H$ is infinite cyclic, $\Phi(A)=\{1\}=\Phi(B)$ and yet $\Phi(G)=H$. In this example both $A, B$ are infinitely generated. The Example 4.1 shows that $\Phi(G)$ behaves "badly" when $A, B$ are finitely generated. Consequently it is rather nice that the following can be obtained.

TheOREM 4.2. Let $A, B$ be finitely generated and $\Phi$-free and suppose $H$ is an infinite cycle. Then $G$ is $\Phi$-free.

Proof. By Theorem $3.1 \Phi(G) \subseteq H$. Thus, if $\Phi(G) \neq\{1\}$, we see that $\Phi(G)$ is infinite cyclic.

Let $X, Y$ denote the centralizers of $\Phi(G)$ in $A, B$ respectively. Clearly $X \supseteq H, Y \supseteq H$ and $|A: X| \leq 2,|B: Y| \leq 2$. Thus $X \triangleleft A, Y \triangleleft B$. Let $X^{\prime}, Y^{\prime}$ be the derived groups of $X, Y$ respectively. Since $\Phi(G)$ is central in $X$ it follows that $\Phi(G) \cap X^{\prime} \subseteq \Phi(X)$. But $X$ is finitely generated and $\Phi(G) \cap X^{\prime} \triangleleft A$ (since $\Phi(G) \triangleleft A$ and $\left.X^{\prime} \operatorname{ch} X \triangleleft A\right)$. Hence $\Phi(G) \cap X^{\prime} \subseteq \Phi(A)$. But $A$ is $\Phi$-free. Hence $\Phi(G) \cap X^{\prime}=\{1\}$; in particular $H \cap X^{\prime}=\{1\}$. Similarly for $Y^{\prime}$ in $B$.

We can thus map $G$ onto $G=\left(\bar{A}^{*} \bar{B}\right)_{H}$, where $\bar{A}=A / X^{\prime}, \bar{B}=B / Y^{\prime}, \bar{H}=H$, under the homomorphism $\psi$, say. Since $\Phi(G) \psi \subseteq \Phi(G)=\Phi(\bar{G})$ we see that $\Phi(\bar{G}) \neq\{1\}$. Now $\bar{X}=X / X^{\prime}, \bar{Y}=Y / Y^{\prime}$ are finitely generated abelian groups 
which we may assume, WLOG, to be aperiodic (for otherwise we can factor out their periodic subgroups $\tau(\bar{X}), \tau(\bar{Y})$ which are normal in $\bar{A}, \bar{B}$ respectively and both of which miss $\bar{H}$ ). Thus we can find bases $x_{1}, \ldots, x_{m}$ of $\bar{X}$ and $y_{1}, \ldots, y_{n}$ of $\bar{Y}$ such that

$$
\bar{H}=\langle h\rangle=\left\langle x_{m}^{s}\right\rangle=\left\langle y_{n}^{t}\right\rangle
$$

for suitable integers $s, t$.

Suppose $\Phi(\bar{G})=\left\langle h^{w}\right\rangle$. Then, for each $a \in A$, we have $a^{-1} h^{w} a=h^{ \pm w}$ (and similarly for each $b \in B$ ). Let $p$ be an odd prime distinct from those dividing $s, t, w$.

Now it is easy to see that $\bar{X}^{p} \cap \bar{H}=\left\langle h^{p}\right\rangle=\bar{Y}^{p} \cap \bar{H}$ and so we may map $\bar{G}$ onto $\overline{\bar{G}}=(\overline{\bar{A}} * \overline{\bar{B}})_{H}$ where

$$
\overline{\bar{A}}=\bar{A} / \bar{X}^{p}, \quad \overline{\bar{B}}=\bar{B} / \bar{Y}^{p}, \quad \overline{\bar{H}}=\bar{H} / \bar{H}^{p} .
$$

Now $\overline{\bar{A}}$ is either a finite elementary abelian group $E_{\mathrm{A}}\left(\right.$ viz. $\bar{X} / \bar{X}^{p}$ ) of exponent $p$ or an extension of $E_{\mathrm{A}}=\bar{X} / \bar{X}^{p}$ by a 2-cycle. Further $\overline{\bar{H}}=\langle\overline{\bar{h}}\rangle$ is a normal ( $p$-cyclic) subgroup of $\overline{\bar{A}}$. Indeed it follows from the remarks above that each element of $\overline{\bar{A}}$ (and likewise of $\overline{\bar{B}}$ ) conjugates the element $\overline{\bar{h}}$ into itself or its inverse. Suppose for the moment that $A$ is not abelian. Then $\overline{\bar{A}}=E_{\mathrm{A}} \cup a E_{\mathrm{A}}$ where $a$ conjugates $\bar{h}$ to its inverse. Consider the set of elements $a^{-1} g a \cdot g$ where $g \in E_{\mathrm{A}}$. Clearly each such element is in $E_{\mathrm{A}}$ (since $E_{\mathrm{A}} \triangleleft \overline{\bar{A}}$ ) and is indeed central in $\overline{\bar{A}}$ (for $E_{\mathrm{A}}$ is abelian whilst

$$
a^{-1}\left(a^{-1} g a \cdot g\right) a=a^{-2} g a^{2} \cdot a^{-1} g a=g \cdot a^{-1} g a=a^{-1} g a \cdot g,
$$

the latter two equalities coming from the fact that $a^{2}, g, a^{-1} g a$ all belong to $E_{\mathrm{A}}$ and hence commute). The set $C_{\mathrm{A}}$ of all such $g^{a} \cdot g$ is a (central) subgroup of $\overline{\bar{A}}$ which misses $\overline{\bar{H}}$ and we can pass to $\left.\overline{\bar{A}} / C_{\mathrm{A}} * \overline{\bar{B}}\right)_{\bar{H}}$. Similar remarks apply to $\overline{\bar{B}}$.

Hence the original $G=\left(A^{*} B\right)_{H}$ has a homomorphic image $G^{*}=\left(A^{*} \times B^{*}\right)_{H^{*}}$ in which $A^{*}, B^{*}$ are each one of the following types of groups: $(\alpha)$ A finite elementary abelian $p$-group $E_{p} ;(\beta)$ An extension of such a group by an element inverting all the elements of $E_{p}$. Further, because of the choice of $p, \Phi\left(G^{*}\right)=H^{*}$ and, since $H^{*}$ is a normal $p$-cycle, bases for the elementary abelian subgroups $E_{\mathrm{A}}, E_{\mathrm{B}}$ can be obtained by extending from a basis for $H^{*}$. In particular if $E_{\mathrm{A}}=\left\langle x_{1}^{*}, \ldots, x_{r}^{*}\right\rangle$ where $H^{*}=\left\langle x_{r}^{*}\right\rangle$ then $A^{*}$ splits over $H^{*}$, a complement being $\left\langle x_{1}^{*}, \ldots, x_{r-1}^{*}\right\rangle$ in case $(\alpha)$ and $\left\langle a, x_{1}^{*}, \ldots, x_{r-1}^{*}\right\rangle$ in case $(\beta)$. Thus in every possible case $A^{*}, B^{*}$ both split over $H^{*}$. Hence $G^{*}$ splits over $H^{*}$ and Lemma 2.6 of [8] tells us $\Phi\left(G^{*}\right) \cap H^{*}=\{1\}$-a contradiction, which completes the proof.

At this point we should like to ask the question whether $\Phi(G)=\{1\}$ for $G=\left(A^{*} B\right)_{H}$ where $H$ is infinite cyclic and $\Phi(A)=\Phi(B)=\{1\}$ with $A$ finitely generated and $B$ infinitely generated. 
5. Applications to knot groups. Neuwirth [7] has asked what can be said about the Frattini subgroup of a knot group. Murasugi conjectures that it is always trivial. This conjecture has recently been proved. ${ }^{(2)}$ However for certain knots the result follows much more easily as we now indicate.

Since a torus knot group has a presentation of the form $\left\langle a, b ; a^{m}=b^{n}\right\rangle$ applying Theorem 3.1 by using $c=b a^{k} b^{-1} a^{-k}$ we immediately have:

THEOREM 5.1. The torus knot groups have trivial Frattini subgroups.

TheOREM 5.2. Let $G=\left(A^{*} B\right)_{\mathbf{H}}$ where $H$ is infinite cyclic and is a retract of $A$ and of $B$. Then $\Phi(G)=\{1\}$.

Proof. By Theorem 2.1, $\Phi(G) \subseteq H$. Now $A=C H, B=D H$, where $C \triangleleft A$, $C \cap H=\{1\}$ and $D \triangleleft B, D \cap H=\{1\}$. Mapping $G$ onto $H$ under $\Theta$, say, by sending $C$ and $D$ onto $\{1\}$ and mapping $H$ identically we see that

$$
\Phi(G) \Theta \subseteq \Phi(G \Theta)=\Phi(H)=\{1\} .
$$

Thus $\Phi(G) \subseteq \operatorname{ker} \Theta$. It follows that $\Phi(G) \subseteq H \cap \operatorname{ker} \Theta$. But it is clear that ker $\Theta \cap H=\{1\}$, whence $\Phi(G)=\{1\}$.

As an immediate consequence we have the following:

THEOREM 5.3 (Murasugi [6]). The knot group of any product of knots has trivial Frattini subgroup.

Proof. The group of product of two knots is the generalized free product of the groups of the constituent knot groups amalgamated along retracts (see [3]), which are infinite cyclic. Hence, by Theorem 5.2 its Frattini subgroup is trivial.

To show that the cable knot has trivial Frattini subgroup we first prove the following lemma.

Lemma 5.4. Let $G=\left(A^{*} B\right)_{H}$. Then $G^{\prime} \cap B=B^{\prime}\left(A^{\prime} \cap H\right)$.

Proof. Clearly $B^{\prime}\left(A^{\prime} \cap H\right) \subseteq G^{\prime} \cap B$, so we only have to prove the reverse inequality. First note that $A_{1}=A^{\prime}\left(B^{\prime} \cap H\right) \triangleleft A, B_{1}=B^{\prime}\left(A^{\prime} \cap H\right) \triangleleft B$ and that $A_{1} \cap H=B_{1} \cap H$. Thus we can map $G$ onto $G_{1}=\left(A / A_{1}{ }^{*} B / B_{1}\right)_{H / A_{1} \cap H}$ cannonically. Since $A / A_{1}$ and $B / B_{1}$ are abelian we can further map $G_{1}$ onto the generalized direct product $G_{2}$, say, of $A / A_{1}$ and $B / B_{1}$ amalgamating $H / H \cap$ $A_{1}$. Since $G_{2}$ is abelian we see that $\operatorname{ker}\left(G \rightarrow G_{2}\right) \subseteq G^{\prime}$. But this kernel meets $A, B$ in exactly $A_{1}$ and $B_{1}$ respectively. Hence $G^{\prime} \cap A \subseteq A_{1}$ and $G^{\prime} \cap B \subseteq B_{1}$, as required.

THEOREM 5.5. Cable knot groups have trivial Frattini subgroups.

\footnotetext{
(2) Murasugi's conjecture has recently been proved by a quite involving topological and group theoretical argument.
} 
Proof. Let $G$ be the knot group of a cable knot. Then $G$ can be put in the form $G=\left(A^{*} B\right)_{H}$ where $B$ is infinite cyclic, and $B \neq H$. By Theorem 2.1, $\Phi(G) \subseteq H$. On the other hand, since $G / G^{\prime}$ is infinite cyclic, it follows that $\Phi(G) \subseteq G^{\prime}$, whence $\Phi(G) \subseteq H \cap G^{\prime}$. If $\Phi(G) \neq 1$ then $B G^{\prime} / G^{\prime}$ must be finite and hence trivial, that is, $B \subseteq G^{\prime}$. Now, by Lemma 5.5 , this is impossible since $B$ is infinite cyclic and $H \varsubsetneqq B$. This proves the theorem.

6. Residually solvable groups. In [9] it was shown that if $G=\left(A^{*} B\right)_{H}$ is residually finite such that $A, B$ each satisfied a nontrivial identical relation which is not satisfied by the infinite dihedral group then $\Phi(G) \subseteq H$. In this section we establish a parallel result for residually solvable generalized free products.

DefintTIOn 6.1. $\left\{S_{i} ; i \in I\right\}$ is called a solvable filter for $G$ if each $S_{i} \triangleleft G$ such that $G / S_{i}$ is solvable and $\bigcap_{i \in I} S_{i}=1$. It is called a solvable $H$-filter if $H$ is a subgroup of $G$ and $\left\{S_{i} ; i \in I\right\}$ is a solvable filter of $G$ such that $\bigcap_{i \in I} S_{i} H=H$.

Defintrion 6.2. Let $G=\left(A^{*} B\right)_{H}$ and $\left\{A_{i} ; u \in I\right\},\left\{B_{i} ; i \in I\right\}$ be solvable filters of $A$ and $B$ respectively, then they are called compatible solvable filters with respect to $H$ if $A_{i} \cap H=B_{i} \cap H$ for $i \in I$.

As it is in the case of residually finite groups, denoted by $R^{\mathscr{F}}$, it can easily be shown that $G=\left(A^{*} B\right)_{H} \in{ }_{R} S$ if $A, B$ have compatible solvable $H$-filters, where ${ }_{R} S$ denotes the class of residually solvable groups.

LEMMA 6.3. Let $G=\left(A^{*} B\right)_{H} \in_{R} S$. If $A, B$ each satisfies an identical relation not satisfied by the infinite dihedral group then $A, B$ have compatible $H$-filters.

The proof of this lemma is similar to that of Theorem 2 of [4].

LeMMA 6.4. Let $G=\left(A^{*} B\right)_{H} \in_{R} S$ and $\delta_{i} G$ be the $i^{\text {th }}$ derived subgroup of $G$. Then $\left\{\delta_{i} G ; i \in J\right\}$, where $J$ is the set of positive integers, is a solvable filter for $G$.

Proof. $G \in_{R} S$ implies there exists a solvable filter $\left\{S_{i} ; i \in I\right\}$. Thus $G / S_{i}$ is solvable and $\bigcap_{i=I} S_{i}=1$. Let $G / S_{i}$ be solvable of length $k$. Then $\delta_{k} G \subseteq S_{i}$. Since $\bigcap_{i \in I} S_{i}=1$ it follows that $\bigcap_{i \in I} \delta_{i} G=1$.

THEOREM 6.5. Let $G=\left(A^{*} B\right)_{H} \in{ }_{R} S$ such that $A, B$ each satisfies a nontrivial identical relation not satisfied by the infinite dihedral group. Then $\Phi(G) \subseteq H$.

Proof. If $|A: H|=|B: H|=2$ then $H \triangleleft G$. Therefore by $[5] \Phi(G) \subseteq H$. Thus we can assume $|A: H| \geq 3$. Since $A, B$ each satisfies an identical relation not satisfied by the infinite dihedral group and $\left\{\delta_{i} G ; i \in J\right\}$ is a solvable filter for $G$, by Lemmas 6.3 and $6.4,\left\{A_{i} ; i \in J\right\}$ and $\left\{B_{i} ; i \in J\right\}$ where $A_{i}=A \cap \delta_{i} G, B_{i}=$ $B \cap \delta_{i} G$, are compatible solvable $H$-filters for $A$ and $B$ respectively. Let 
$\bar{A}_{i}=A / A_{i}$ and $\bar{B}_{i}=B / B_{i}$. Since $A_{i}$ and $B_{i}$ are compatible, we have $A_{i} \cap H=$ $B_{i} \cap H=H_{i}$. Hence we can form $\bar{G}_{i}=\left(\bar{A}_{i}^{*} \bar{B}_{i}\right)_{\bar{H}_{i}}$, where $\bar{H}_{i}=H A_{i} / A_{i}=H B_{i} / B_{i}$. Moreover $A_{1} \subsetneq A, B_{1} \subsetneq B$ and $\bigcap_{i \in J} A_{i} H=\bigcap_{i \in J} B_{i} H=H$ it follows that $\bar{G}_{i}$ is a proper generalized free product. Since $|A: H| \geq 3,\left|\bar{A}_{i}: \bar{H}_{i}\right| \geq 3$. Thus $G_{i}$ is not an infinite dihedral group. Hence $\bar{G}_{i}$ is not solvable.

Let $G_{i}=G / \delta_{i} G$. Then $G_{i}$ is solvable of length $i$. On the other hand $G_{i}=$ $\left\langle A \delta_{i} G / \delta_{i} G, B \delta_{i} G / \delta_{i} G\right\rangle$. Now,

$$
\frac{A \delta_{i} G}{\delta_{i} G} \approx \frac{A}{A \delta_{i} G}=\frac{A}{A_{i}}=\bar{A}_{i} .
$$

In the same way $B \delta_{i} G / \delta_{i} G \approx \bar{B}_{i}$. Hence ther exists a natural homomorphism $\rho_{i}: \bar{G}_{i} \rightarrow G_{i}$ such that $\left.\rho_{i}\right|_{\bar{A}_{i}},\left.\rho_{i}\right|_{\bar{B}_{i}}$ are the natural isomorphisms between $\bar{A}_{i}$ and $A_{i} \delta_{i} G / \delta_{i} G$ respectively. Clearly, $\operatorname{ker} \rho_{i} \cap \bar{H}_{i}=1$. Since $G_{i}$ is solvable while $\bar{G}_{i}$ is not solvable, it follows that $\operatorname{ker} \rho_{i} \neq 1$. Hence, by Theorem 2.1 [2], $\Phi\left(\bar{G}_{i}\right) \subseteq \bar{H}_{i}$ for each $i$.

Suppose $\Phi(G) \not \subset H$. Then there exists $a \in A \backslash H$ and $a \in \Phi(G)$. (See proof of Theorem $2.1[1])$. Now $\left\{A_{i} ; I \in J\right\}$ is an $H$-filter. Therefore there exists an $i$ such that $a \notin A_{i} H$. Let $\psi_{i}$ be the natural homomorphism of $G$ onto $\bar{G}_{i}$. Then $a \psi_{i} \notin \bar{H}_{i}$. But $a \psi_{i} \in \Phi\left(\bar{G}_{i}\right) \subseteq \bar{H}_{i}-$ a contradiction. Hence $\Phi(G) \subseteq H$. This proves the theorem.

It can be noted that the method of the proof of Theorem 6.5 is applicable to Theorem 3.5(i), (ii) [9] while the converse is not true. In fact the method of the proof of theorem of Theorem 6.5 can be applied to any $G=\left(A^{*} B\right)_{H} \in{ }_{R} P$, where $P$ is any property $P$, such that the $P$-filter $\left\{N_{i} ; i \in I\right\}$ of $G$ satisfies the property that $G / N_{i} \cap N_{j}$ is again $P$. Thus we can make a more general statement:

TheOREM 6.6. Let $G=\left(A^{*} B\right)_{H} \in{ }_{R} P$ such that $A, B$ each satisfies a nontrivial identical relation not satisfied by the infinite dihedral group. Then $\Phi(G) \subseteq H$.

\section{REFERENCES}

1. R. B. J. T. Allenby and C. Y. Tang, On the Frattini subgroup of a residually finite generalized free product, Proc. Amer. Math. Soc., 47 (1975), 300-304.

2. R. B. J. T. Allenby and C. Y. Tang, On the Frattini subgroups of generalized free products and the embedding of amalgams, Trans. Amer. Math. Soc., 203 (1975), 319-330.

3. James Boler and Benny Evans, The free product of residually finite groups amalgamated along retracts is residually finite, Proc. Amer. Math. Soc., 37 (1973) 50-52.

4. R. J. Gregorac, On residually finite generalized free products, Proc. Amer. Math. Soc., 24 (1970) 553-555.

5. G. Higman and B. H. Neumann, On two questions of Ito, J. London Math. Soc., 29 (1954) 84-88.

6. K. Murasugi, Lecture notes on knot theory, Univ. of Toronto, 1971.

7. L. P. Neuwirth, Knot groups, Princeton University Press, 1965.

8. C. Y. Tang, On the Frattini subgroups of generalized free products with cyclic amalgamations, Canad. Math. Bull., 15 (1972), 569-573. 
9. C. Y. Tang, On the Frattini subgroups of certain generalized free products of groups, Proc. Amer. Math. Soc., 37 (1973), 63-68.

10 A. Whittemore, On the Frattini subgroup, Trans. Amer. Math. Soc., 141 (1969), 323-333.

SCHOOL OF MATHEMATICS,

UNIVERSITY OF LEEDS,

LEEDS, ENGLAND.

Department of Pure Mathematics,

UNIVERSITY OF WATERLOO,

WATERLOO, ONTARIO, N2L 3G1

DEPARTMENT OF MATHEMATICS,

SAN Francisco State University,

CALIFORNIA, U.S.A. 